\title{
Study of Multimedia Technology in Posture Training for the Elderly
}

\author{
Chih-Chen Chen ${ }^{1}$, De-Jou Hong ${ }^{2}$, Shih-Ching Chen ${ }^{3}$, Ying-Ying Shih ${ }^{4}$, Yu-Luen Chen ${ }^{2,5}$ \\ ${ }^{1}$ Department of Management Information System, Hwa Hsia Institute of Technology, Taipei, Chinese Taipei \\ ${ }^{2}$ Department of Computer Science, National Taipei University of Education, Taipei, Chinese Taipei \\ ${ }^{3}$ Department of Physical Medicine \& Rehabilitation, Taipei Medical University Hospital, Taipei, Chinese Taipei \\ ${ }^{4}$ Department Physical \& Rehabilitation Medicine, Chang Gung Memorial Hospital, Tao-Yuan, Chinese Taipei \\ ${ }^{5}$ Department of Information Management, St. Mary’s Medicine, Nursing and Management College, Yilan, Chinese Taipei \\ Email: weib120@gmail.com, sheau2767@gmail.com, allen@tea.ntue.edu.tw
}

Received October 2012

\begin{abstract}
Wrist diseases, also known as "mama hand", "mouse hand" or "keyboard hand", are commonly seen and easily overlooked symptoms in daily life. The diseases are mostly related to incorrect exercise or excessive force imposed on hands, leading to tenosynovitis or carpal tunnel syndrome. To alleviate the symptoms or even to recover, besides drug treatment, supplementary rehabilitation training is necessary. The rehabilitation process forces the affected wrist area to continually exercise in order to stimulate the self-repair signals sent to the affected area for partial restoration, if not all, of the supposed functions. In modern world, using information technology to improve the rehabilitation environment with better approaches has become a trend. In this study, software programs coded in C\# and Flash are developed to work on the Bluetooth ball hardware to facilitate the rehabilitation. The basic principle is to use the reaction from the Bluetooth ball's swaying or swinging to drive the movement of the objects on the computer screen, making the supposedly boring rehabilitation much more fun and vivid in the interactive multimedia environment, thus gaining better treatment effect. Also, RFID, Internet and databases can be integrated into this facility to provide patient identification and data storage for references in making rehabilitation training programs.
\end{abstract}

Keywords: Rehabilitation; Bluetooth Ball; RFID; Databases; Interactive Multimedia

\section{Preface}

The interactive multimedia controller has evolved rapidly, from the early knob and joystick to the Xbox360 controller, the PS3 controller's direction button and press button, and then the Wii remote controller. The controller design has changed from the two-hand gripping to the single handheld rod, coupled with the triaxial acceleration detection technology to determine the action of the hand swaying and infrared optical positioning technology to detect the direction of the controller's front end, for the interface control.

This study makes an insight into the rehabilitation training aided with the Bluetooth ball for signals transmission. The numeric values generated by the Bluetooth ball actions drive the movement of the objects on the computer screen, making the supposedly boring rehabilitation much more fun and vivid, for a better medical result. And the rehabilitation data can be integrated into the RFID patient identification, Internet access connection, computer server storage, and database queries, to constitute a comprehensive management mechanism for medi- cal references.

Of all the wrist diseases, the "mama hand", whose scientific name is stenosing tenosynovitis, occurs at age 30 to 50 , and female's incidence is about 10 times that of male. It's the stenotic tendon bursitis of the extended thumb brevis tendon and longus tendon of the thumb abduction near the side of wrist, which brings tendon glide difficulties and causes wrist pain. However, the "mouse hand" and "keyboard hand" are of the carpal tunnel syndrome, which is caused by the wrist transverse ligament pressing the wrist median nerve, occurring at age 30 to 60 to those who repeat their wrist exercises over and over, and statistics shows the male and female incidence ratio is $1: 4$. Also, using mobile phones continuously for a long time can bring great pressure to the palm, causing wrist pains in many cases. To alleviate the symptom or even recover from the disease, besides drug treatment, complimentary rehabilitation is necessary.

\section{Literature Review}

Bluetooth ball was created by Finland Ball-It [1], since it 
has been massively used in entertainment. Following are some of the literatures on its applications.

In his article titled "Interactive multimedia applications-illustrated with the campus navigation system", Chau-Cheng Cheung argues that the merit of Bluetooth ball wireless transmission is to get rid of the keyboard and mouse for control, and instead use the handheld Bluetooth ball to do pinch, press, toss, and rotation to send instruction signals to Quest3D for corresponding actions to control the objects in the scene, making the campus navigation system more flexible and fun [2].

Yu-Jie Lin, in his article titled "A hand rehabilitation system based on Bluetooth ball: case studies of stroke disabled adults," points out that the rehabilitation system built on Bluetooth ball games can increase stoke patients' interest in rehabilitation. The Bluetooth ball games presented on the computer screen can increase the visual stimulation, which appears very helpful in the interference phase and helps the participants understand their own rehabilitation statues. Besides, the visual and audio combination gets patients' attention and makes them more interested in rehabilitation [3].

In his paper titled "The development of traceable intelligent weighing system" released for an industrial-university cooperative research project, Bai-Sheng Chen suggests a mechanism that can automatically integrate consumers' health information (e.g. height, weight, BMI, eating habit, exercise habit), calories of consumer food (e.g. beverages, light food, fruit) and its traceability system, health maintenance recommendations, and shopping recommendations, which are processed by the triaxial six-directional wireless Bluetooth ball to calculate the calorie consumption in exercise, so as to fulfill the goals of food traceability and health management [4].

Chin-Jui Chang et al. in the article "Applications for medical recovery using wireless control of a Bluetooth ball with a hybrid G-sensor and human-computer interface technology" point out that extension of the Bluetooth applications helps the development of human-machine interfaced interactive games which allow players to follow the rhythm of songs to change the hand pressing actions or the speed of swaying the Bluetooth ball, so as to improve the flexibility of the hand movement and enhance the body balancing [5].

\section{The Research Methods and Purposes}

\subsection{Wrist Diseases}

The commonly seen wrist diseases are mama hand, mouse hand and keyboard hand. When housewives cook, they use hands to grip the pot handle and turning shovel, and move their wrists up and down, and such a persistent movement can hurt the wrist and cause the mama hand disease. When working on a computer, one will use fin- gers to click the mouse or two hands to strike the keyboard, with the wrist posed in a fixed position for a long duration, and such a persistent movement day after day can finally cause serious damage to the wrist. Also, riding motorcycle with a fixed hand position to turn the throttle can also cause keyboard hand disease after a long-term operation.

\subsection{System Planning}

Figure 1 shows the schematic overview of the study. Before using the Bluetooth system, following preprocesses are required:

- The hardware:

This includes an RFID reader and tag, a PC with a Bluetooth device, and a Bluetooth ball (make sure it is adequately charged and can communicate with the PC via the Bluetooth). Since Bluetooth ball stays in sleep mode under constant state, we need to wake it up by tossing it, shaking is or swaying it. On the system startup, the ball's blue light will flash.

- The software:

Microsoft Visual Studio C\# 2008, Microsoft SQL Server 2008, Adobe Flash CS4. Visual Studio C\# constitutes the main program codes, and SQL Server is responsible for managing the database that stores the RFID card numbers, user information and the Bluetooth ball-related data. As for Flash CS4, it supports the Action Script 3.0 syntax and the Flash file is played under Visual Studio C\#.

After all the preparation is done, we can start to execute the program. To begin with, it is the RFID authentication, during which the RFID tag is detected by the reader which then displays the tag number and sent it to the SQL Server to search for a matched tag number and its holder information stored in the database; if the RFID holder is an administrator, the administrator's screen will

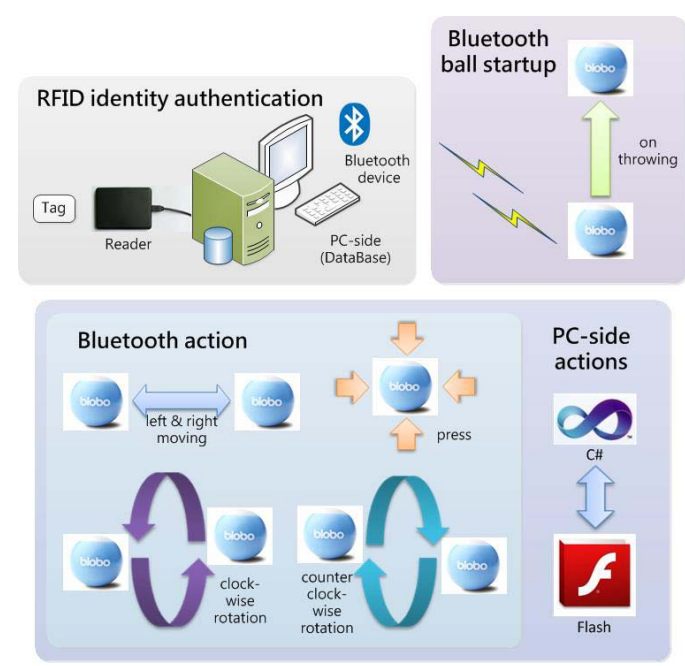

Figure 1. Overall schematic diagram. 
be displayed, and if the holder is a general user, the user training screen will be displayed. Before doing the exercise, toss up the Bluetooth ball to start it, then connect the $\mathrm{PC}$ to the Bluetooth and test the communication by moving the ball left and right in the hand for the ball's built-in electronic gyroscope, pressure sensor and GSensor to detect the $\mathrm{X}$-axis acceleration values. When the exercise is done, the wireless communication between the PC and the Bluetooth ball will terminate, and X-axis acceleration values resulted from the exercise will be averaged and stored in the SQL Server database for retrieval later on when one wants to resume previous exercise so the averaged $\mathrm{X}$-axis acceleration value can be applied directly to the gaming screen.

The gaming mechanism is schemed into two parts: the Bluetooth ball movement and the PC end reactions. The former part includes moving left and right, press, clockwise and counterclockwise rotations which are respectively reacted in the latter part PC-end screen display of moving left, right, up and down, and rotation. Before the game is started, play the Flash file under the Visual Studio C\# execution first, and then proceed to connect the PC with the Bluetooth ball so that the ball can do its work, which is correspondingly reacted on the PC screen. After the game is done, the communication between the PC and the Bluetooth ball will terminate, and the data collected during the gaming period will be stored in the SQL Server database and can be used for analysis on the training effect.

\subsection{Process Flow Design}

- The hardware: connection between the PC and Bluetooth ball:

Figure 2 demonstrates the flow of connection between the PC and Bluetooth ball, and the connection is based on the Bluetooth communication protocol. Bluetooth ball is in sleep mode when it stays still. So, toss the ball up or heavily shake it to wake it up before using it. Once the ball is initiated, it is in on status, ready for subsequent connection to the PC. The ball will seek available connection devices, and if the connection fails, it could be insufficient charge of the ball; if so, recharge the ball and have it seek connection spots again.

- The software:

This is categorized into preprocessing of database creation, identity authentication and training activities.

1) Identity authentication

The flow chart of identity authentication shown in Figure 3 is to determine the identity of an administrator or a user. The authentication is done by matching the RFID tag number which is unique to an individual user, and is required for entry into the program. If the identity is determined as an administrator, the maintenance mechanism for managing the electronic tag numbers and

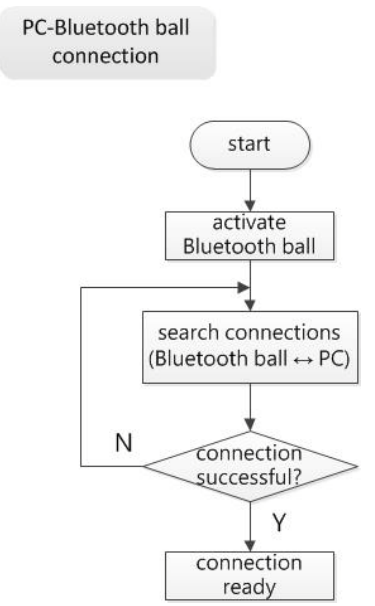

Figure 2. The flowchart of PC and Bluetooth ball.

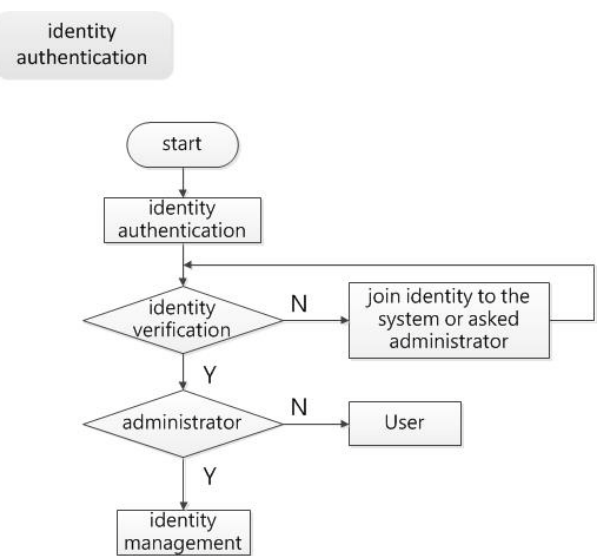

Figure 3. The flow chart of identity authentication.

user identities in the database is enabled; if the tag number is absent in the database, an authentication failure message will be displayed and adding a new tag number to the database is required so that the new user can proceed with the operations.

2) Training activities

The overall flow chart of the training activities shown in Figure 4 can be divided into the practice screen and gaming screen. After being authenticated, the user enters the practice screen. Since every user has his/her unique hand acceleration force, before getting to the formal exercise, the user is required to do the acceleration force practice and test. This is done by holding the Bluetooth ball for a specified duration and continuously swinging it left and right for it to capture the acceleration values in the right and left directions (X-axis); the values are then averaged and stored in the database. One of the games designed in this study is Tetris, which has graphic boxes in different shapes on the screen for the player to rearrange them into appropriate positions, and the player scores when a bunch of boxes are seamlessly put together into a row, and the row is then erased. Later on, when the 

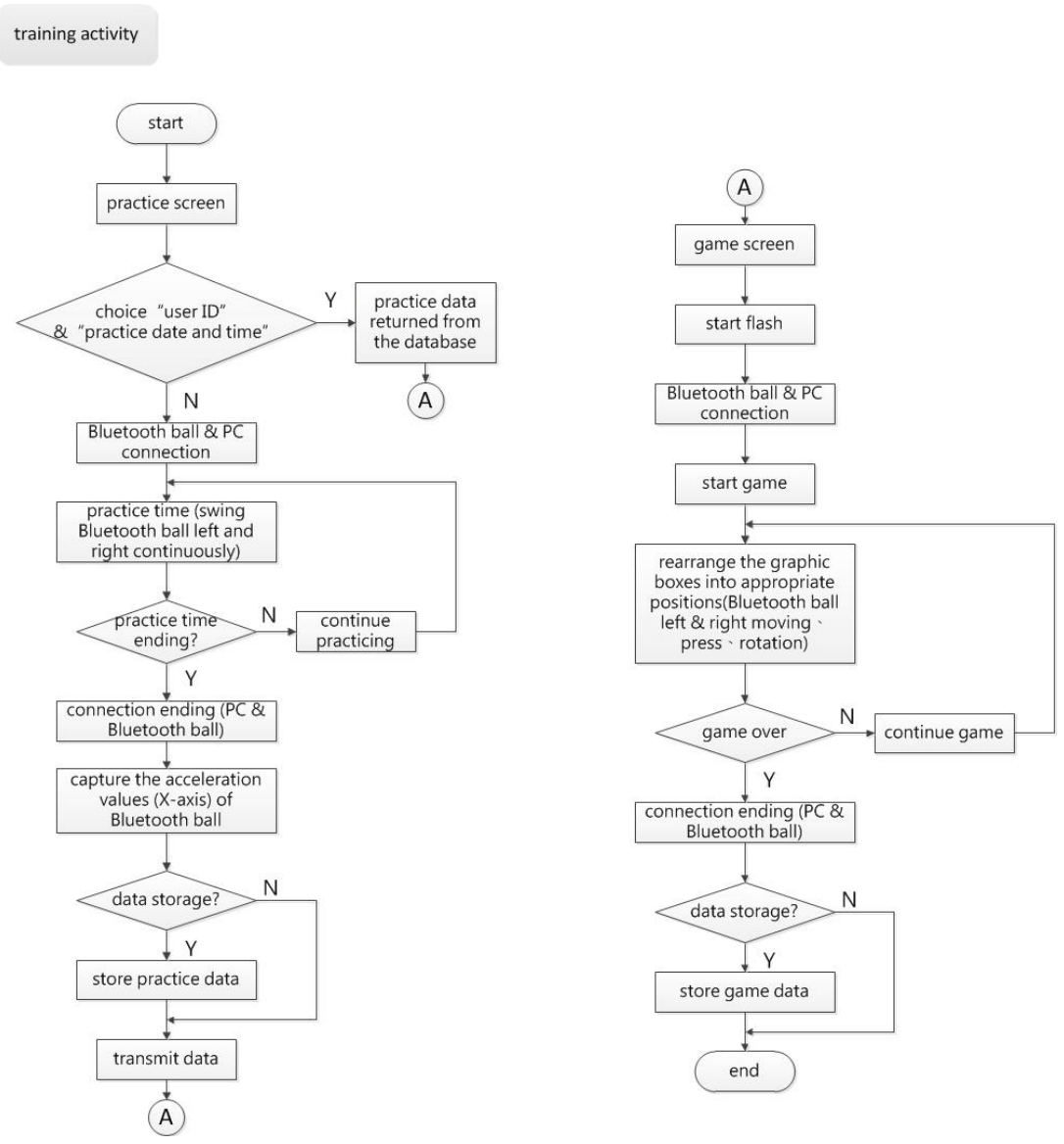

Figure 4. Overall flow chart.

patient in training re-enters the gaming screen, the patient's acceleration force data is first retrieved from the database and passed to the gaming screen. Before the game begins, the Flash needs to be initiated and then the PC and the Bluetooth ball be connected for communication. The Bluetooth movements are left and right moves, press and rotation, which are correspondingly reacted on the screen as the manipulated object's left and right, up and down moves and rotation. When the gaming is finished, the connection between the PC and Bluetooth ball terminates, and the time used as well as the scores gained during the gaming session are stored in the database and can be used later on for rehabilitation analysis and program planning.

\section{Results and Discussion}

The purpose of this study is to introduce the multimedia interactive technology to the wrist rehabilitation training programs, in the hope of presenting an interesting and convenient wrist rehabilitation approach.

- Identity authentication

Figure 5 shows the RFID authentication screen. The RFID reader detects the tag number and sends it to the database for identity matching authentication.

- The practice screen

Figure 6 shows the practice screen. The user holds the Bluetooth ball in his hand and sways it left and right, and the system fetches the ball's X-axis acceleration values in a fixed time interval. When the practice is done, the user's averaged acceleration values in the left and right directions are calculated for later use in the formal gaming exercise.

- The gaming screen

One of the games developed in this study is Tetris as shown in Figure 7. Originally, Tetris was played with the keyboard. The new Tetris developed in this study is manipulated with the electronic gyroscope, pressure sensor and G-Sensor inside the Bluetooth ball to make wrist rehabilitation much more effective and fun as well

- The empirical test

In this study, a middle-aged woman suffered from minor mama hand is taken as an empirical test subject (see Figure 8). The training is given 3 times a week, with each time taking 10 minutes, for a total training period of 3 weeks. In this training, the Bluetooth ball's left and right moves, rotation and press are given to manipulate the Tetris game. Of the manipulative moves, the left and 


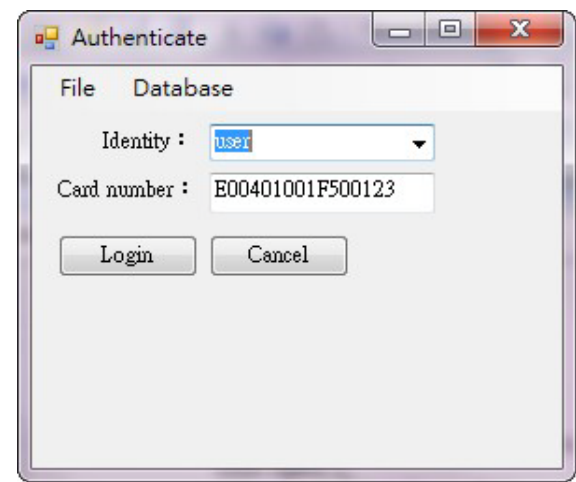

Figure 5. Identity authentication.

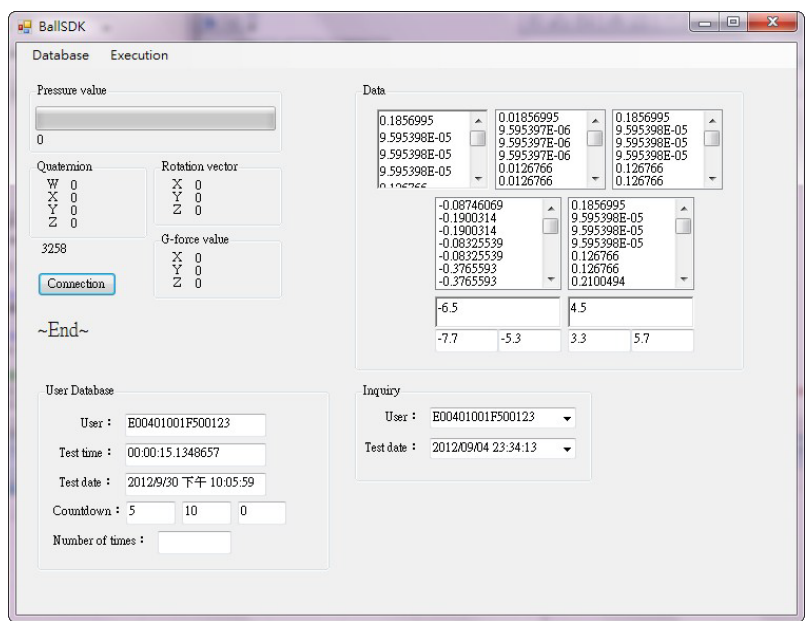

Figure 6. Practice screen.

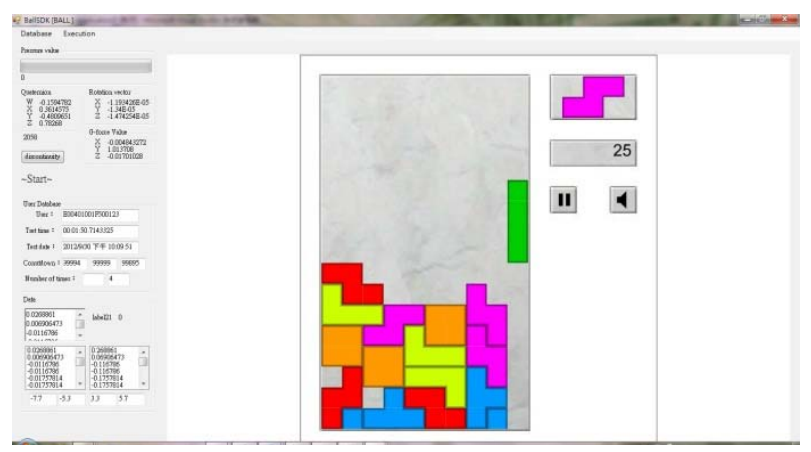

Figure 7. Game screen.

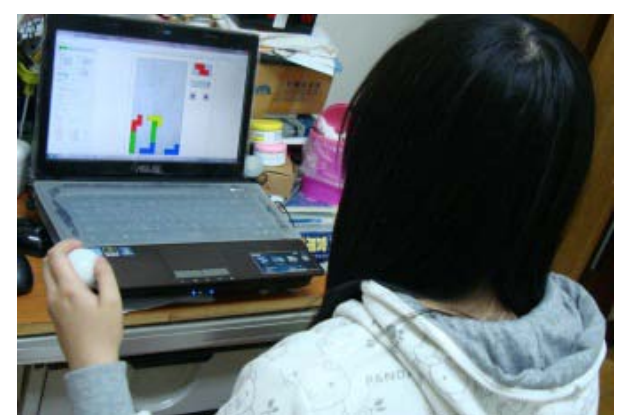

Figure 8. Empirical test. right moves command horizontal shifts of the square to the left and right; the rotation flips the square over; the press action drops the square quickly. It is a 25-point gain when a bunch of boxes are put together into a seamless row and the row will then be erased. The score will be accumulated as more rows are completed. During the gaming, if any of the squares is stacked up to touch the top of the screen, the game is over. If the training time is not up yet, click the game again to re-enter the gaming screen and continue the training.

The result of the training on this subject is shown in Figure 9. The red box indicates the game is over and the number in the red box stands for the score. The empirical analysis shows that the subject was not familiar with the use of the Bluetooth ball in the 3 practices of the first week, causing repetitive restarts of the game, and gaining no score in the first 2 practices, but gaining 25 points in the 3rd practice-an indication the subject was getting more familiar with the game. Starting from the 2nd week, however, the game restart became less frequent, and the scores were getting higher. In the 3rd week, there was no restart of the game in the 3rd practice. The results reveal how this woman played the game and how well she was getting more familiar with the use of the Bluetooth ball. Also, the less game restarts and the higher scores demonstrate the rehabilitation was getting its performance into effect.

\section{Conclusion}

In this study, the Bluetooth wireless communication technology is applied to the medical rehabilitation practices. In contrast with the traditional rehabilitation approach that is compulsive exercise-centric, monotonous and unable to automatically record and analyze the rehabilitation data, the interactive multimedia-aided rehabilitation developed in this study offers versatility, vividness and mobility, bringing lots of fun to the supposedly boring and long-lasting rehabilitation process. Furthermore,

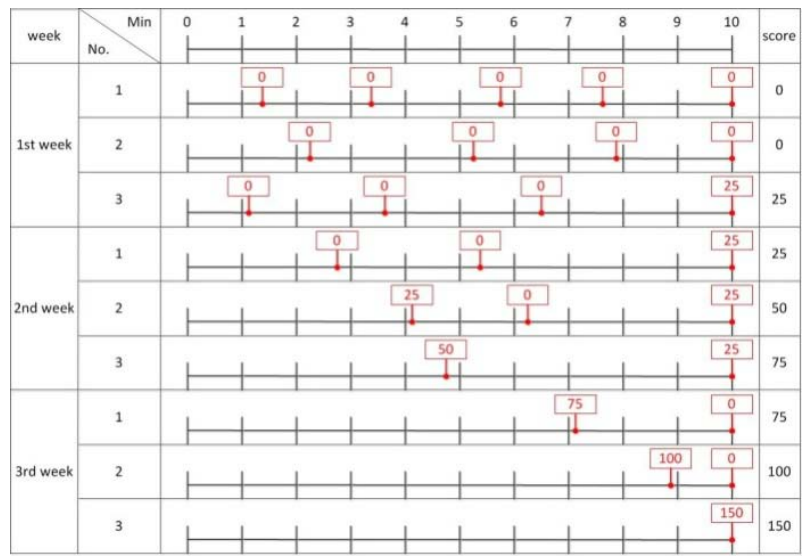

Figure 9. Training results. 
the RFID authentication technology makes it possible to store the rehabilitation data, such as the rehabilitation dates and times used in the exercises, which can then be used for analyses. This makes breakthrough for the traditional approach, thus increases the rehabilitation effects.

\section{Acknowledgements}

The authors wish to thank the National Science Council (NSC) in Taiwan (Grant Number: NSC 101-2221-E-146001-, NSC101-2221-E-152-001-, NSC101-2627-E-002006-) for support this research.

\section{REFERENCES}

[1] Ball-It. http://www.ball-it.com/index.html

[2] C.-C. Cheung, "Interactive Multimedia Applications-
Illustrated with the Campus Navigation System,” Master Thesis, Nan Jeon Institute of Technology, 2011.

[3] Y.-J. Lin, "A Hand Rehabilitation System Based on Bluetooth Ball: Case Studies of Stroke Disabled Adults,” 2012.

[4] B.-S. Chen, "The Development of Traceable Intelligent Weighing System,” A Concise Report on the Outcomes of the Industry, University Cooperative Research Program Subsidied by the National Science Council of the Executive Yuan, The Technology and Knowledge Application Type, 2010.

[5] C.-J. Chang, C.-Y. Chen and C.-W. Huang, “Applications for Medical Recovery Using Wireless Control of a Bluetooth Ball with a Hybrid G-Sensor and Human-Computer Interface Technology,” Journal of Vibration and Control, Vol. 19, No. 8, 2013, pp. 1139-1151. 